\title{
The Geochemistry of Coral Biomineralization: Using Boron Isotopes to Understand the Cost of Resilience in a Changing Ocean
}

ALEXANDER C. GAGNON ${ }^{1 *}$, ANNE M. GOTHMANN ${ }^{1,2,3}$, OSCAR BRANSON ${ }^{4}$, JAMES W. B. RAE ${ }^{5}$, AND JOSEPH A. STEWART $^{6}$

${ }^{1}$ School of Oceanography, University of Washington, Seattle, WA, USA (*correspondence: gagnon@uw.edu)

${ }^{2}$ Joint Institute for the Study of the Atmosphere and Ocean, University of Washington, Seattle, WA, USA.

${ }^{3}$ Departments of Physics and Environmental Studies, St. Olaf College, Northfield, MN, USA.

${ }^{4}$ Department of Earth Sciences, University of Cambridge, Cambridge, UK.

${ }^{5}$ School of Earth and Environmental Science, University of St. Andrews, St. Andrews, Scotland.

${ }^{6}$ School of Earth Sciences, University of Bristol, Bristol, UK

Coral calcification is both sensitive to environmental changes and buffered from these changes though the physiology of biomineralization. The mechanisms governing this balance determine how corals respond to ocean acidification and control the sensitivity of many paleoclimate proxies. One such promising proxy in corals and other marine calcifiers is skeletal boron isotopes, which are used to reconstruct ocean $\mathrm{pH}$ changes over geologic time. Because boron isotopes reflect calcifying fluid chemistry, understanding how boron responds to a wide range of $\mathrm{CO}_{2}$ chemistry conditions can improve this paleoclimate proxy while also elucidating environmental controls on biomineralization. We show that skeletal boron isotopes are not a simple indicator of seawater $\mathrm{pH}$ but are also sensitive to the total dissolved inorganic carbon (DIC) concentration of seawater in the cultured cold-water coral Balanophyllia elegans. A general geochemical model of calcification, which includes boric acid diffusion across cell membranes, accurately predicts both this new DIC effect and the behavior of the boron paleo-pH proxy. Contrary to previous studies, our results demonstrate that skeletal boron isotopes do not necessarily reflect calcifying fluid $\mathrm{pH}$, which remains nearly constant in the species studied here. This constant calcifying fluid $\mathrm{pH}$ implies similar calcification rates during ocean acidification, which may explain the resilience of B. elegans to ocean acidification. Nevertheless, this resilience comes at an energetic cost because our model shows that calcification becomes less efficient as seawater becomes more acidic. 